\title{
Technical refinements of the modified central mound breast reduction
}

\author{
Simon Nicholson', Muhammad Adil Abbas Khan², Muhammad Riaz ${ }^{3}$ \\ ${ }^{1}$ Hand Surgery Fellow, Middlemore Hospital, Otahuhu, Auckland 2025, New Zealand. \\ ${ }^{2}$ ST6 Registrar in plastic surgery, NHS Tayside, Dundee DD2 1GZ, UK. \\ ${ }^{3} \mathrm{Hull}$ and East Riding Hospital, Anlaby, Kingston upon Hull HU10 7AZ, UK.
}

Correspondence to: Dr. Simon Nicholson, Hand Surgery Fellow, Middlemore Hospital, Otahuhu, Auckland 2025, New Zealand.

E-mail: plasticandhandsurgeon@gmail.com

How to cite this article: Nicholson S, Khan MAA, Riaz M. Technical refinements of the modified central mound breast reduction. Plast Aesthet Res 2018;5:34. http://dx.doi.org/10.20517/2347-9264.2018.30

Received: 28 Apr 2018 First Decision: 16 Jul 2018 Revised: 10 Aug 2018 Accepted: 20 Aug 2018 Published: 21 Sep 2018

Science Editor: Raúl González-García Copy Editor: Yuan-Li Wang Production Editor: Zhong-Yu Guo

\begin{abstract}
Aim: Common pitfalls with existing breast reduction techniques include poor aesthetic outcome, such as development of a "boxy" breast shape, and pseudoptosis. Presented here are a series of modifications to the technique of central mound breast reduction, based on previous work, aimed at ensuring consistent aesthetic results which are maintained in the long-term.
\end{abstract}

Methods: All patients undergoing bilateral breast reduction by the senior author over a 7-year period were included, with outcome data collected prospectively. A detailed description of the technique is offered.

Results: One hundred and sixteen patients underwent bilateral breast reduction over the study period. Mean followup was 20.6 months. There were no cases of nipple necrosis or infection requiring antibiotics. There was one postoperative haematoma which required surgical evacuation. Three patients developed a degree of fat necrosis which was managed conservatively in two, but required surgical debridement for liquefactive necrosis in one. Results of these breast reductions at the second post-operative year and beyond are presented.

Conclusion: The technique described offers benefits of improved predictability, consistency and longevity of aesthetic results over existing techniques. Development of pseudoptosis in particular is effectively delayed. The modifications described have not been shown to increase the rates of surgical complications.

Keywords: Central, mound, breast, reduction, mastopexy, technique, technical, refinement, modification

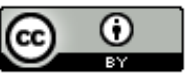

(C) The Author(s) 2018. Open Access This article is licensed under a Creative Commons Attribution 4.0 International License (https://creativecommons.org/licenses/by/4.0/), which permits unrestricted use sharing, adaptation, distribution and reproduction in any medium or format, for any purpose, even commercially, as long as you give appropriate credit to the original author(s) and the source, provide a link to the Creative Commons license, and indicate if changes were made.

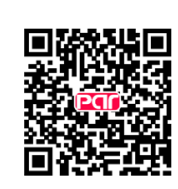




\section{INTRODUCTION}

Breast reduction has been described and modified by over 30 authors over more than 100 years ${ }^{[1]}$, and most techniques share the common pitfalls of pseudoptosis (bottoming out), difficulty ensuring an aesthetically pleasing shape, and failure to maintain aesthetic results in the long-term.

Central mound breast reduction, in which the gland with the nipple at its apex contains the pedicle of blood supply to the nipple, is reported to maintain nipple sensation well ${ }^{[2]}$. In addition it maintains a reliable blood supply and allows good visualisation of the whole gland for shaping and removal of tissue.

We describe a series of refinements to the technique of central mound breast reduction, designed to optimise the aesthetic outcome and maintain longevity of results. This technique can be applied to reductions of large or small breast volumes, or of simple mastopexy without reduction.

\section{Principles of this technique are as follows}

A smooth conical breast shape is obtained by carefully contouring the gland during the reduction, suspending and wrapping the gland from below using a dermal sling, before re-draping the widely undermined skin envelope.

A wider breast base is created by elevating the gland beyond the normal superior limit.

To reduce pseudoptosis the gland is plicated inferiorly using part of the dermal sling, which acts to shorten the distance between the areola and the inframammary fold (IMF), such that the skin envelope is not relied upon for support of the gland.

The gland is maintained in its new elevated position on the chest wall by a series of anchor points between the inferior dermal sling, an additional superior dermal strip, and the rib periosteum.

The IMF is secured to the chest wall in order to maintain lower pole definition, further reduce tension on the skin envelope, and further reduce the effects of gravity on recurrent ptosis.

The latter three points ensure no additional tension on the skin envelope, beyond that necessary for closure, when the patient is upright. The effect of gravity on the skin is therefore diminished.

In large heavy breasts the footprint or base of the breast is narrow, so it is necessary to elevate the skin envelope beyond the normal superior limit of existing breast base \& extend it close to the clavicle. This creates a reduced breast with a wider base which "takes off" more superiorly than the usual point where a ptotic breast starts.

In breast ptosis, the IMF can "slide" down the chest wall along with the rest of the base of the breast, independently of any increase in IMF to nipple-areola-complex (NAC) distance. An incision parallel and 2-3 $\mathrm{mm}$ superior to the IMF line bevelled in a superior direction aims to preserve and protect the IMF ligament. If this ligament is secure already, it should be kept intact. If it has become attenuated and the IMF separates from the chest wall, as is the case in patients who lose large volumes of weight, it is often readily noticeable and should be identified and addressed during the procedure.

\section{METHODS}

All patients undergoing bilateral breast reduction by the senior author (M.R.) in both public and private practices over a 7-year period were included. Data on patient demographics, tissue mass excised, and post- 


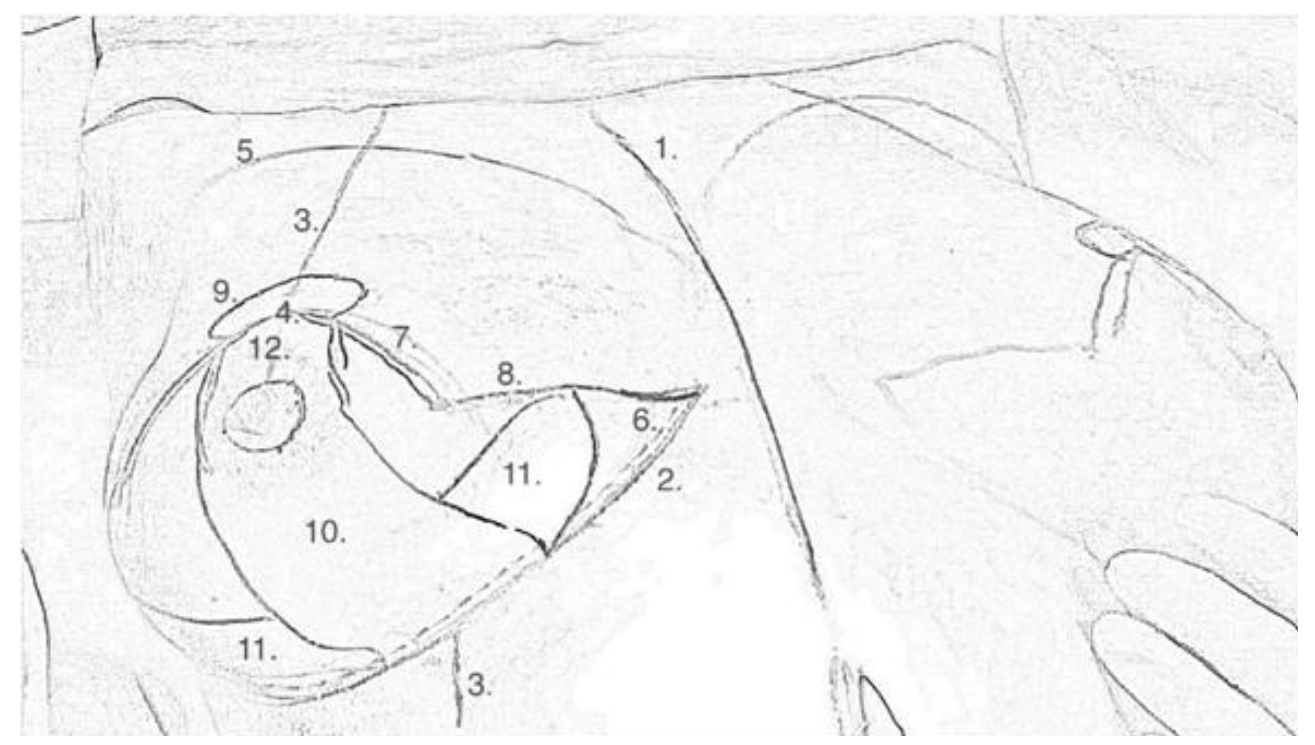

Figure 1. Skin markings. See body of text for number key

operative complications was collected prospectively and stored by the senior author in a secure database. All those included in the study received a minimum of 6-month follow-up. The surgical technique evolved gradually over the preceding decade, including incorporation of previous authors' work and is detailed as follows. The case examples shown have given consent for publication of their anonymised images.

Preliminary skin markings are made with the patient upright and are later re-drawn with the patient supine, after the operative field is prepared and draped [Figure 1]. Markings are similar to those for an inferior pedicle wise pattern technique, but with the addition of medial, lateral and superior dermal wings. Markings therefore consist of: (1) vertical midline from suprasternal notch to xiphoid process; (2) IMFs; (3) breast meridians (usually using a tape measure draped around the patient's neck) continuing through the areola and onto the inframammary chest wall; (4) intended height of the new nipple based on Pitanguy's point; and (5) the superior margin of the breast mound. The new nipple height is measured bilaterally to confirm symmetry.

Another line is drawn parallel and 2-3 mm superior to the IMF mark (6), which represents the line of fullthickness dermal incision. The narrow strip between here and the IMF is de-epithelialised, to which the IMF retaining sutures are later anchored.

The vertical edges of the skin envelope are marked as lines running $7.5 \mathrm{~cm}$ from the centre of the new nipple position in a caudal direction (7), while rotating and displac-ing the gland clockwise and anticlockwise. The lower limits of these lines are then turned $90^{\circ}$ towards the ends of the IMF mark (8). The angle the vertical lines make where they meet at the new nipple position is determined by measuring the length of the $\mathrm{IMF}^{[2]}$ and the corresponding lines of the skin envelope (8) to ensure the overall length is the same. If the IMF is longer than the combined length of the horizontal parts of the skin envelope, the angle at which the vertical lines meet is narrowed. A broken circle (9), greater in width than height, of around $4.5 \mathrm{~cm}$ diameter is marked, centred on the intended new nipple position. The exact diameter can be determined by patient preference.

A vertical dermal strip roughly $7 \mathrm{~cm}$ in width is marked, equivalent to that of an inferior pedicle breast reduction (10). Near the base of this strip, a wing-like projection is also marked on each side, with about $5 \mathrm{~cm}$ 


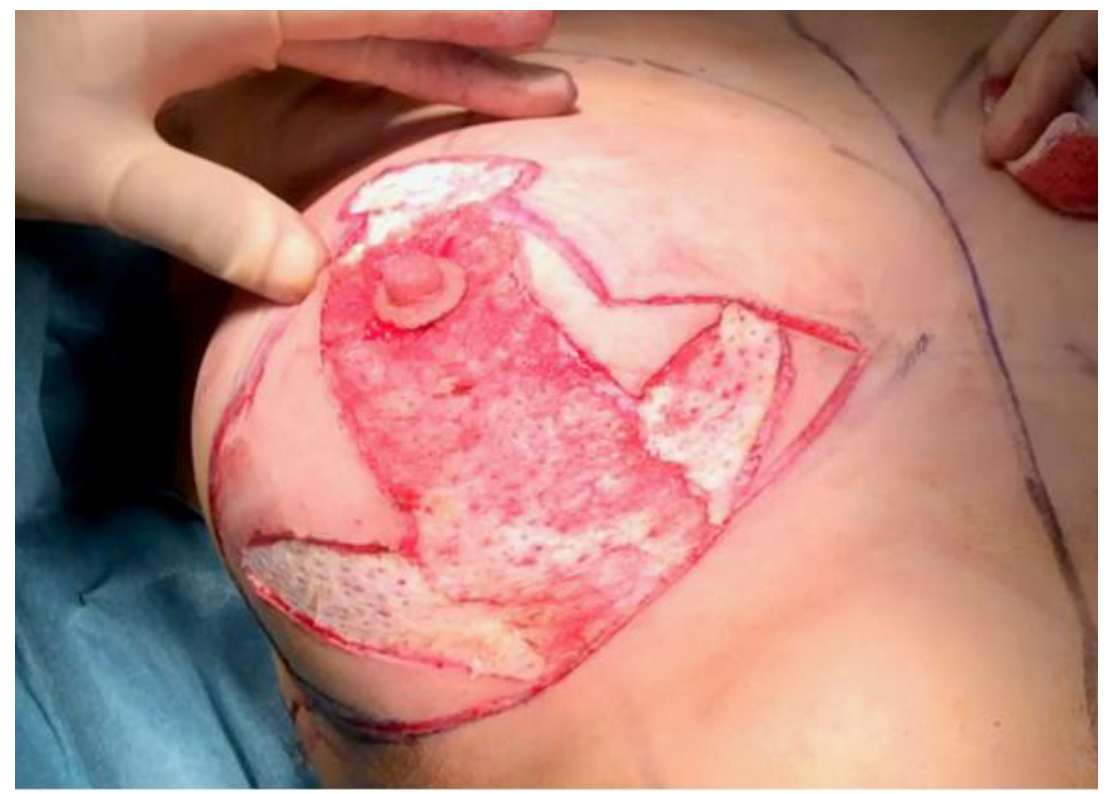

Figure 2. De-epithelialised dermal strip including one superior and two lateral wing-like extensions

base width and a length sufficient to wrap around the side of the glandular mound to create a conical shape in later steps (11). A third dermal wing arises from the superior aspect of the vertical dermal strip, above the nipple, within the broken circle at the top of the markings (12). The length of the vertical dermal strip below the nipple is often much longer (and variable between individuals) than the distance from the nipple to the IMF when the skin envelope is closed. This strip will be plicated to reduce it's length, by an amount that varies from person to person, and help prevent pseudoptosis in the long term.

The skin envelope is infiltrated with a solution containing local anaesthetic and adrenaline, with emphasis laterally and superiorly, to block intercostal nerve sensory branches and constrict the intercostal perforator vessels. The smaller breast is reduced first. All of the marks are scored. Without the use of a breast tourniquet, the vertical dermal strip, along with the 3 dermal "wings", are de-epithelialised using handswitch monopolar, sparing the nipple [Figure 2]. The incision parallel to the IMF is beveled superiorly to keep the ligamentous attachment of IMF intact, and prevent the gland descending postoperatively.

Full thickness incisions into the underlying subcutaneous tissue commence with elevation of the medial dermal wing at a thickness of around $1 \mathrm{~cm}$. The skin at the medial end of the IMF scar is undermined while an assistant provides elevation with skin hooks [Figure 3]; the volume from this area is reduced to avoid a dog-ear. The same is then done laterally.

Laterally the tissue to be excised is raised, leaving fibrofatty tissue on the chest wall of a thickness corresponding to the layer of subcutaneous fat. This avoids injury to neurovascular structures and contour irregularity when the skin envelope is closed.

Skin flaps are raised starting medially. The assistant uses skin hooks in the breast tissue (not dermis) and lifts the tissue vertically away from the chest [Figure 4]. Dissec-tion with handswitch monopolar proceeds in the plane of fascia is often referred to as the mastectomy plane, or sometimes deeper; the desired thickness of flaps is around $2 \mathrm{~cm}$. One hand is used to keep checking the thickness of the flap. The medial skin flap is not fully raised at this time. 


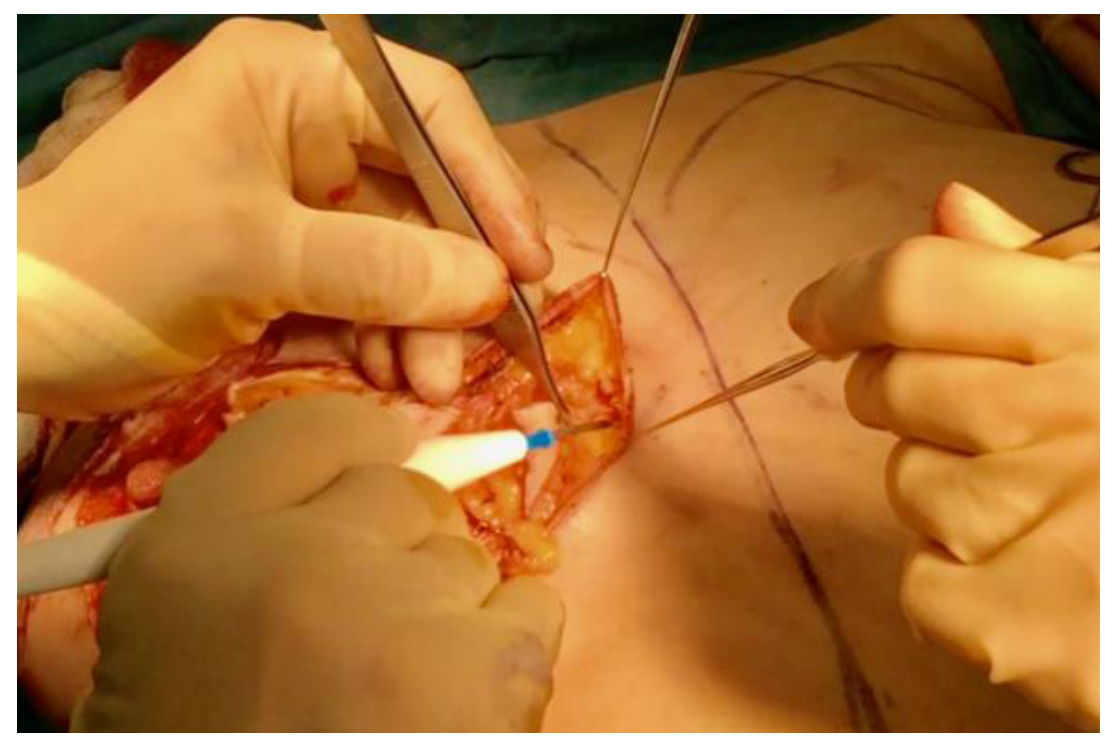

Figure 3. Undermining of the skin at the medial end of the inframammary fold (IMF), to allow tension-free re-draping of the skin at closure and prevent standing cone deformity

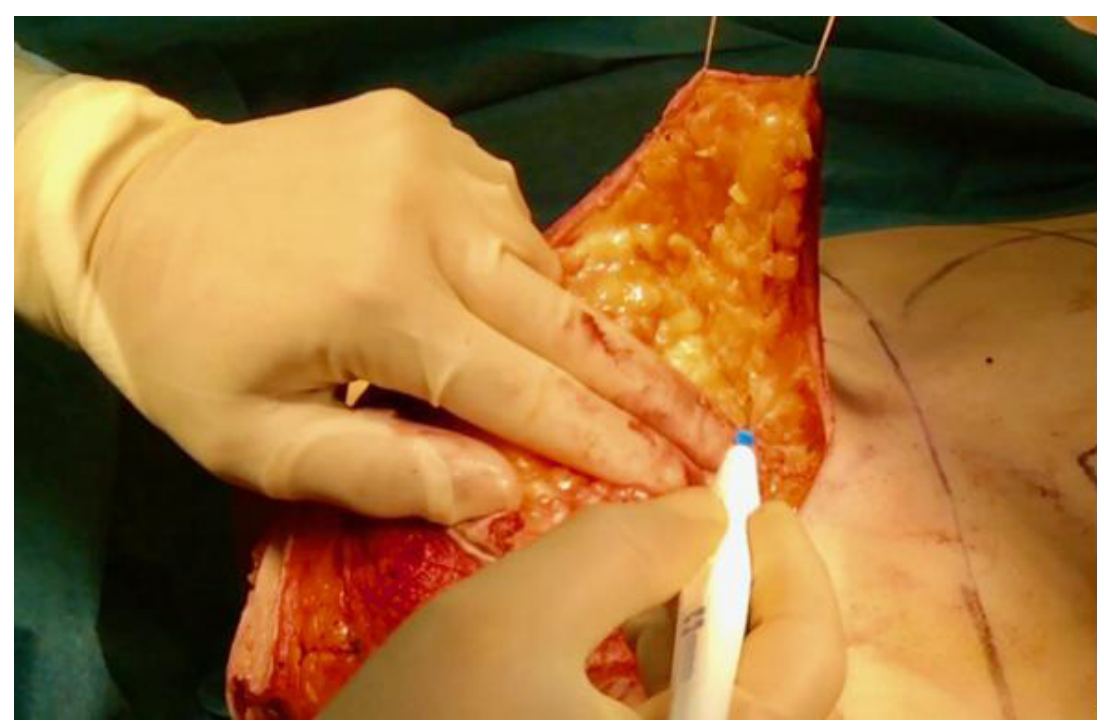

Figure 4. Elevation of the medial flap of the skin envelope, with assistant retracting away from the chest using skin hooks

The lateral flap is raised in the same way and at the same thickness, again not completely. The superior part of the skin envelope is elevated beyond the existing superior border of the breast base to create a pocket almost close to the clavicle, as planned preoperatively, to widen the base and to create fullness of upper pole. Adequacy of undermining is checked by placing a hand in the pocket formed by the skin envelope. The superior part is then connected to the medial and lateral parts of the skin envelope flap.

The reduction is then performed. Tissue that is not part of the skin flaps or the central mound, as defined by the dermal strip and lateral wings, is excised. This usually corresponds to the tissue beneath the small triangles of skin remaining around the already dissected parts. Tissue is preserved on the chest wall at the lower medial and lateral quadrants and at the superior pole to ensure good contour. Meticulous attention is paid to haemostasis with the patient at normotension. 


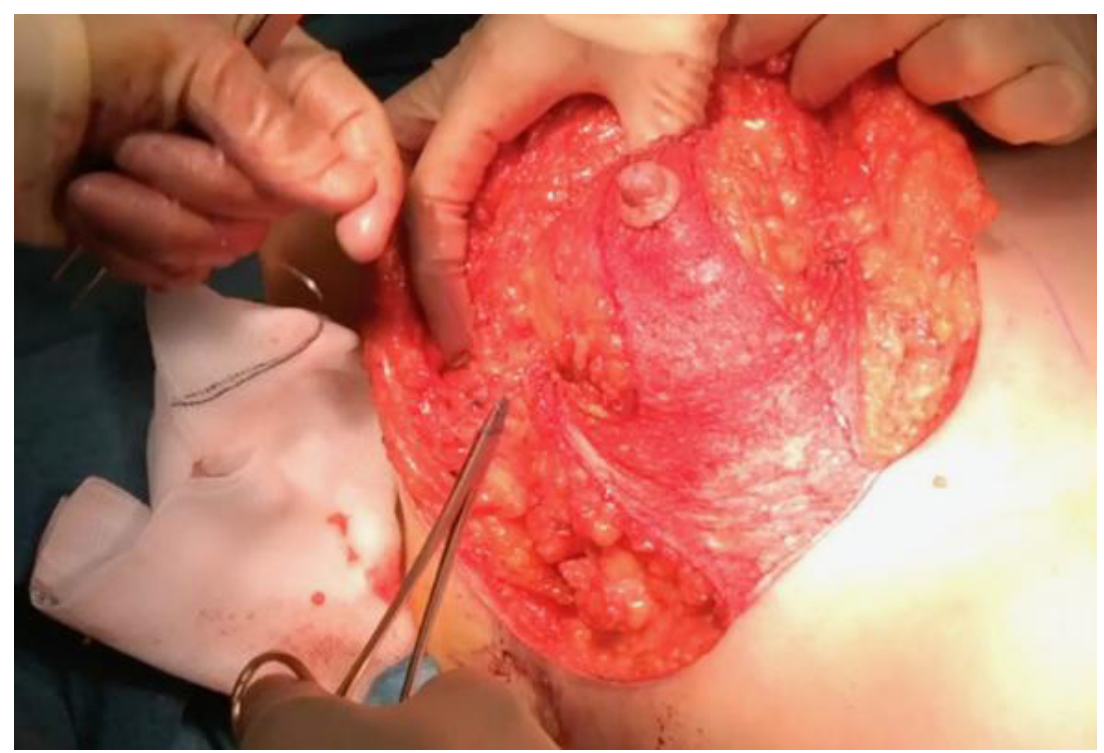

Figure 5. Creation of a conical breast mound and hitching the gland to an elevated position on the chest wall by suturing dermal wings to rib periosteum in a superior direction

The essential dermoglandular suspension consists of a 2-0 prolene suture from the chest wall - either rib periosteum or the pectoralis fascia at the upper aspect of the undermined skin flap - to the superior aspect of the central mound, roughly half way from the base to the superior edge of the de-epithelialised vertical strip. It is tied loosely to avoid tissue ischaemia. With the same suture, a further bite is taken at the superior part of the de-epithelialised dermal strip and tied just tight enough to elevate the nipple to its new height. Thus the mound is sutured to a new higher position on the chest wall. Again, this is not tied tightly so as to avoid restricting the plication of the inferior part of the dermal strip later.

The dermal wings are sutured to the chest wall in a superior direction, wrapping partly around the central mound with 2-0 prolene [Figure 5]. If sutured at an adequate height on the chest, the IMF is lifted slightly, and the overall effect of wrapping the dermal wings around the gland is to create a youthful cone-shaped breast mound for the skin envelope to be re-draped over.

To enhance this cone shape, the supero-medial aspect of the medial and lateral dermal wings are sutured to the sides of the NAC, and the infero-lateral aspect of the wings are sutured to the chest wall.

The nipple is then partially inset using 3-0 vicryl, with a suture superiorly, inferiorly, then medially and laterally.

The necessary plication of the inferior dermal strip is performed using 3-0 Polydioxanone [Figure 6]. The distance from the nipple to IMF along the dermal strip is reduced to a length that corresponds to the vertical edge of the skin envelope and the desired nipple-to-IMF distance.

If required, the IMF ligament is reinforced or reconstructed by suturing the dermis at the IMF, particularly at the corner of the vertical dermal strip, to the chest wall using 2-0 ethibond. Latterly we have used additional de-epithelialised dermal flaps based inferiorly at the IMF incision as anchors for strength of the sutures between the IMF and chest wall. Suturing between dermis and periosteum or pectoralis fascia is more robust than suturing into the fibrofatty tissue around the IMF. 


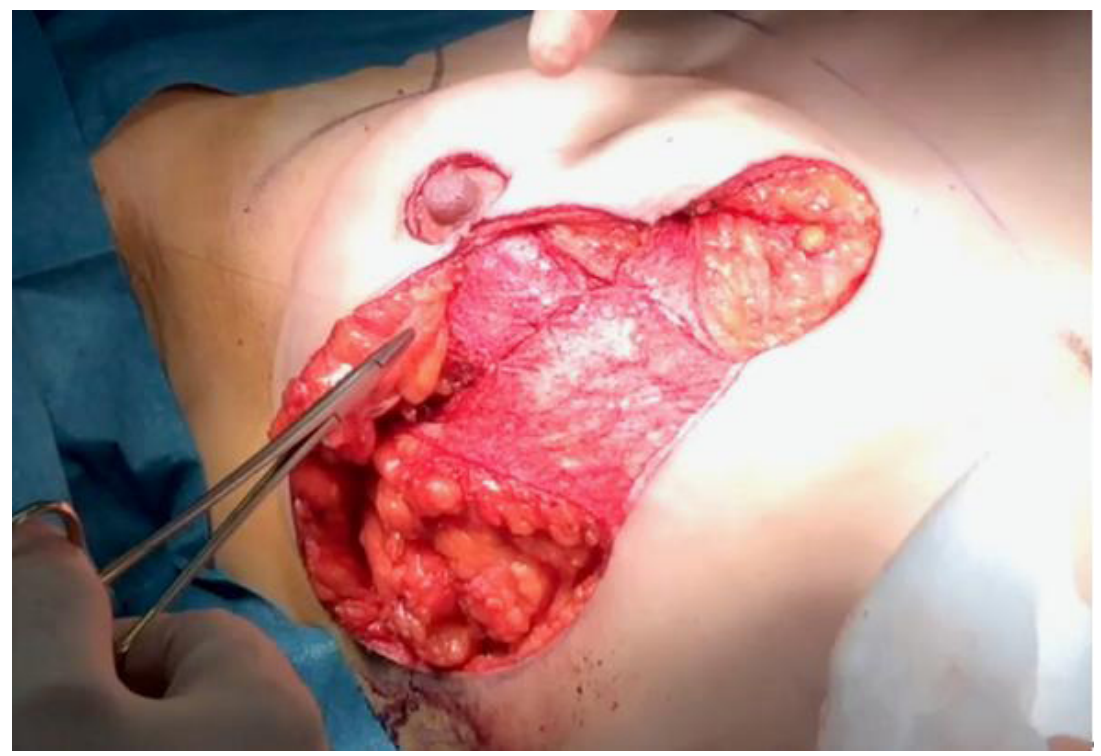

Figure 6. Plication of the inferior dermal strip to shorten the distance between the nipple and the inframammary fold

When closing the skin envelope, 2-0 prolene sutures are placed from the fascial layer to near the IMF to occlude the lateral space at the side of the gland, to prevent the tissue from lateralising and a boxy appearance of the breast. These sutures are also key to shaping the breast base, and some may be used at the medial aspect as well. The skin envelope is closed in the usual way without drains. Occasionally, volume may need to be removed from the ends of the IMF wound before final closure to prevent standing cone deformities. No formal wound drainage is required. Dressings are applied and a sports bra is provided for the patient to wear for 2 months.

\section{RESULTS}

Our series consists of 116 patients, of mean age 40 years (range 19-65). Patients were from a range of racial backgrounds and exhibited broad differences in breast history including previous breastfeeding and breast surgery. The mean tissue mass excised was $865 \mathrm{~g}$ (range: skin only-2175 g) per breast. There were no cases of nipple necrosis or infection requiring antibiotics. There was one post-operative haematoma which required surgical evacuation. Three patients developed a degree of fat necrosis which was managed conservatively in two, but required surgical debridement for liquefactive necrosis in one. Four patients experienced superficial wound dehiscence at the T-junction which was managed successfully with dressings.

Mean follow up was 20.6 months. Several patients treated with this technique are now at post-operative year seven, and none have required revision surgery for correction of pseudoptosis or other recurrent deformities. We did not note any apparent differences in scar quality with this technique compared to those obtained before we began using the technique, though this was not formally assessed. There was no notable difference in breast firmness after surgery with this technique. The effects on lactation were not assessed, though we do not expect these technical modifications to have more of an impact than other breast reduction techniques, and it is possible that the impact may be smaller due to this being a central mound technique.

Typical cases are shown in Figures 7-9. Note the fullness at the upper poles shortly after surgery in Figure 7. By three months this fullness has now descended along with the rest of the breast, including the IMF to a natural non-ptotic shape. The effects of surgery are well-maintained at discharge at 2 and 3 years post-op. 

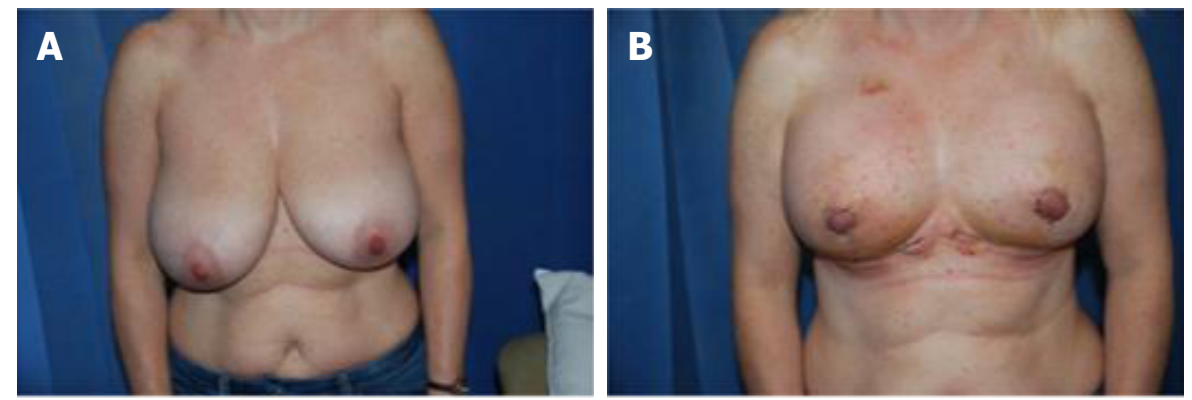

Figure 7. A: Pre-operative; B: ten-day post-operative
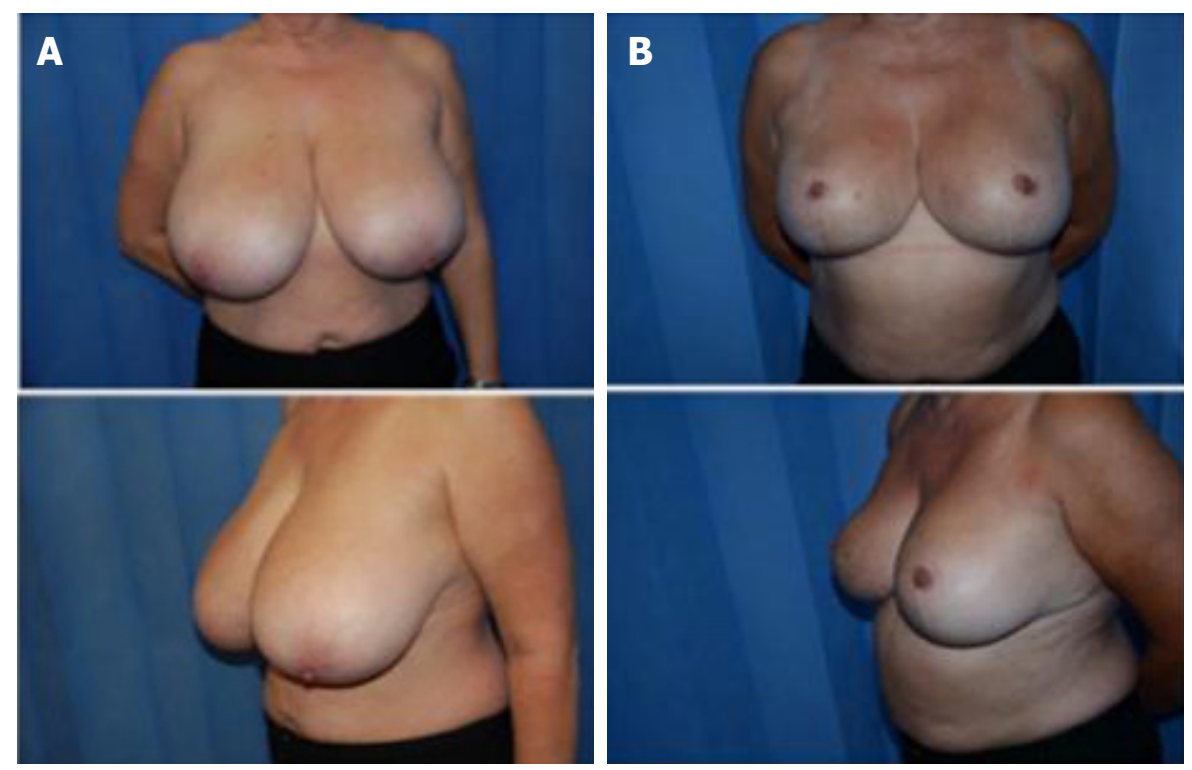

Figure 8. A: Pre-operative; B: three-year post-operative

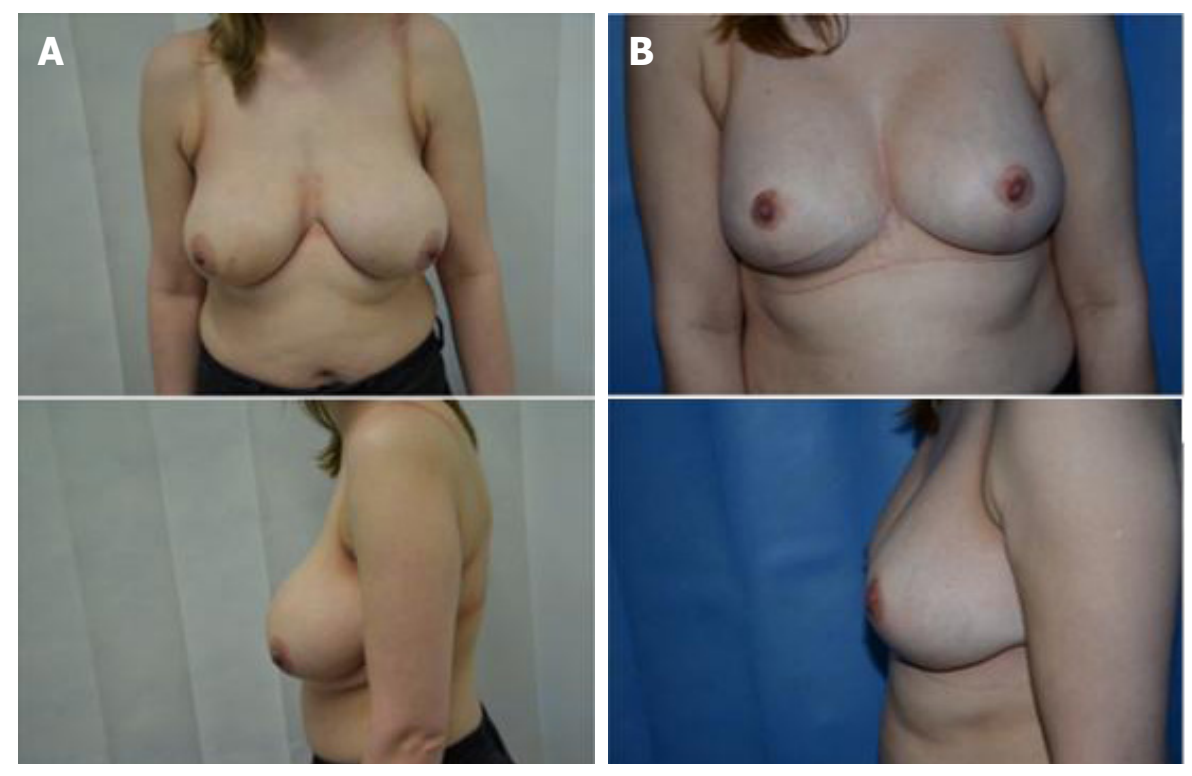

Figure 9. A: Pre-operative; B: two-year post-operative 


\section{DISCUSSION}

Kankaya et al. ${ }^{[3]}$ state that "it is generally accepted that bottoming out deformity starts to develop at approximately six months postoperative", and can be noticed easily at the end of the first postoperative year. Taking this into consideration, our follow-up interval seems appropriate given that many patients do not want unnecessary routine follow-up appointments once their treatment has ended. Our technique reliably reduces the appearance of the bottomingout deformity at postoperative year one and beyond. Although not as extensive as some other studies ${ }^{[4]}$, the number of patients in this series was adequate to demonstrate the consistency of results as seen in the photographs.

Aydin et al..$^{[5]}$ describe creating an internal bra effect by the use of dermoglandular suspension beneath the separate skin envelope. Several variants on this technique exist, which include using alloplastic or allogenic materials ${ }^{[6]}$. Our technique is another variant on similar pre-existing descriptions ${ }^{[7,8]}$ wherein we rely solely on the patient's autologous tissue, which has benefits of not increasing cost, and eliminating the risks associated with implanted materials.

Rubin's paper on mastopexy in the massive weight loss patient ${ }^{[9]}$ refers to plication of the dermis at the lower pole, so as to reduce the distance between the nipple and IMF with the aim of taking tension off the skin envelope at closure and improving projection. Kankaya et al. ${ }^{[3]}$ believe the superior dermo-glandular hitching is sufficient and that inferior dermal plication is not required. We make use of inferior dermal plication in the present technique in the belief that it reduces the propensity for bottomingout. Another purpose of it is to reduce wound healing complications by reducing tension on the skin at closure.

Rubin $^{[9]}$ also makes use of dermo-glandular flaps to create a precisely shaped conical breast mound and suspend the breast tissue on the chest wall. He continues dissection of the skin envelope superiorly beyond the superior limit of the gland up as high as the clavicle. We create a similar pocket but just above the second rib.

Kankaya et al. ${ }^{[3]}$ assert that fixation of the dermal flaps to the costal periosteum in-stead of pectoral fascia can decrease tissue descent over the long term, and Rubin ${ }^{[9]}$ advocates this also. We favour fixation to rib periosteum in our technique wherever possible.

A factor often overlooked in studies of breast reduction is the stability of the IMF. Interference with the normal anatomy of the IMF by surgery can result in the fold being displaced in any direction, including away from the chest wall. If this occurs there is loss of the natural definition of the lower breast pole, and the parenchyma can more readily descend on the chest wall resulting in pseudoptosis or an inferior "double bubble" deformity ${ }^{[10]}$. As part of our technique we place emphasis on dissection superior to the IMF ligament, as defined by Bayati and Seckel ${ }^{[10]}$, to maintain stability of the IMF. The fold may be moved slightly in a superior direction as part of the dermal suspension element of the procedure, but this adds to its definition and does not interfere with the IMF ligament itself. Recently we have also begun to augment the definition of the IMF by adding extra non-absorbable sutures between the dermis and the chest wall with promising initial results. This, we believe, also stabilises the IMF to the chest wall to prevent it from separating or descending.

With ageing, the normal footprint of the breast becomes narrowed due to a combination of ptosis and tissue atrophy. The heavier a breast, the greater the descent due to gravity. Restoration of a youthful breast appearance should involve correction of this senescent narrowing, in addition to correcting ptosis. Our technique creates a dermoglandular cone which, on hitching upward and stabilising to the pectoralis fascia to maintain the mound above the IMF, broadens the footprint of the breast leading to a smooth, round pleasing breast appearance. 
Drawbacks to our technique mainly pertain to the dissection of the skin envelope and the suspension elements: the series of modifications we use in combination add time to the breast reduction overall, of the order of around $30 \%$, but we believe the benefits of improved aesthetics and longevity make this a worthwhile trade-off.

The large skin envelope is widely undermined and relatively thin compared to other techniques. Logically, this introduces a hypothetically increased risk of skin flap necrosis or wound healing complications such as dehiscence or poor scars, particularly at the t-junction. So far we have not yet seen this borne out in practice. Our rates of wound healing complications are comparable to those previously reported elsewhere. Meticulous attention to flap thickness and tissue handling, plus patient selection, may help ameliorate these risks. The skin flap dissection, among other elements of the technique, produces a learning curve that may deter others from incorporating these modifications into their breast reduction repertoire.

Most of our patients wish to keep good proportionate volume ranging from $\mathrm{C}$ to $\mathrm{F}$ cup. As always, we aim to match the breast volume to the body structure of each individual patient in our population. However those wishing to keep a large proportion of initial volume but obtain good breast shape and correction of ptosis, as is possible with this technique, are the challenging patients in the long run due to the rate of recurrent ptosis or pseudoptosis.

The case that developed fat necrosis requiring surgical washout and debridement had a reduction in nippleto-IMF distance of $19 \mathrm{~cm}$. Other cases in our series have had reductions in nipple-to-IMF distance of 17 $\mathrm{cm}$ done safely without complications. We therefore feel it is safe to reduce the pre-operative nipple-to-IMF distance by up to $17 \mathrm{~cm}$ without free nipple graft, assuming intrinsic patient factors are optimal.

In conclusion, the technique herein described offers a range of benefits over existing techniques, aimed at improving predictability, consistency and longevity of aesthetic results. Development of pseudoptosis in particular, which is a common pitfall with existing breast reduction techniques, is effectively delayed. We believe the learning curve and extra time required to complete the procedure, with its series of additional steps, is a very worthwhile trade-off for patients who receive a more lasting improvement in breast shape and lift. The modifications described have not been shown to increase the rates of surgical complications.

\section{DECLARATIONS}

\section{Authors' contributions}

Concept, design and data analysis: Riaz M, Nicholson S

Data acquisition: Riaz M, Khan MAA

Manuscript preparation, critical revision and finalizing of the manuscript: Nicholson S, Riaz M, Khan MAA

\section{Availability of data and materials}

The data were strictly obtained from medical records according to the privacy policy and ethics code of our institute.

Financial support and sponsorship None.

\section{Conflicts of interest}

All authors declared that there are no conflicts of interest.

\section{Ethical approval and consent to participate}

Approval from hospital trust audit department. Full consent from all patients obtained. 


\section{Consent for publication}

Not applicable.

\section{Copyright}

(c) The Author(s) 2018.

\section{REFERENCES}

1. Shiffman, Melvin A. Mastopexy and breast reduction: history of breast reduction. Berlin: Springer-Verlag; 2009. p. 251-2.

2. See MH. Central pedicle reduction mammoplasty: a reliable technique. Gland Surg 2014;3:51-4.

3. Kankaya Y, Oruç M, Sungur N, Aslan ÖÇ, Gürsoy K, Özer K, Koçer U. Four flap suspension technique for prevention of bottoming out after breast reduction. Ann Surg Treat Res 2016;90:10-5.

4. Pennington DG. Improving the results of inferior pedicle breast reduction using pedicle suspension and plication. Aesthetic Plast Surg 2006;30:390-4.

5. Aydin H, Tümerdem B, Onel D, Cerçi Ozkan A. Reduction mammaplasty using inferior pedicle technique combined with dermal suspension. Plast Reconstr Surg 2003;111:1362-3.

6. Góes JC. Periareolar mastopexy: double skin technique with mesh support. Aesthet Surg J 2003;23:129-35.

7. Pérez-Macias JM. Dermis suspension in mammoplasty. Br J Plast Surg 1999;52:421.

8. de Araujo Cerqueira A. Mammoplasty: breast fixation with dermoglandular mono upper pedicle flap under the pectoralis muscle. Aesthetic Plast Surg 1998;22:276-83.

9. Rubin JP. Mastopexy after massive weight loss: dermal suspension and total paren-chymal reshaping. Aesthet Surg J 2006;26:214-22.

10. Bayati S, Seckel BR. Inframammary crease ligament. Plast Reconstr Surg 1995;95:501-8. 